\title{
256.
}

\section{ON A SYSTEM OF ALGEBRAIC EQUATIONS.}

[From the Philosophical Magazine, vol. xx. (1860), pp. 341, 342.]

THE determination of $a, b, c$ from the system of equations

$$
\begin{aligned}
& a^{2}+b c=\lambda, \\
& b^{2}+c a=\mu, \\
& c^{2}+a b=\nu,
\end{aligned}
$$

in the case where $\lambda, \mu, \nu$ have the values 16,17 , and 18 respectively, is the problem known as Colonel Titus's Arithmetical Problem. See Masères' Tracts on the Resolution of affected Algebraic Equations, Lond. 1800. If for shortness we put

$$
\sigma=\nu-c^{2},
$$

then the third equation gives $b=\frac{\sigma}{a}$; and substituting this value of $b$ in the two other equations, we have

$$
\begin{aligned}
& a^{2}+\frac{\sigma c}{a}=\lambda, \\
& \frac{\sigma^{2}}{a^{2}}+c a=\mu ;
\end{aligned}
$$

or what is the same thing,

$$
\begin{aligned}
a^{3}-\lambda a+\sigma c & =0, \\
c a^{3}-\mu a^{2}+\sigma^{2} & =0 ;
\end{aligned}
$$

and from these equations, eliminating $a$, we have

$$
\sigma^{4}-3 c^{2} \sigma^{3}+\left(3 c^{4}-2 \lambda \mu\right) \sigma^{2}-c^{2}\left(c^{4}-\lambda \mu\right) \sigma+c^{4} \lambda \mu-c^{2}\left(\lambda^{3}+\mu^{3}\right)+\lambda^{2} \mu^{2}=0,
$$


where $\sigma=\nu-c^{2}$. The equation in $c^{2}$ is thus of the fourth order; and in like manner, if instead of $c^{2}$ we take $\sigma$ as the unknown quantity, and substitute therefore for $c^{2}$ its value $\nu-\sigma$, the equation in $\sigma$ will be also of the fourth order: and effecting the reduction, this equation is

$$
8 \sigma^{4}-12 \nu \sigma^{3}+\left(6 \nu^{2}-2 \lambda \mu\right) \sigma^{2}+\left(\lambda^{3}+\mu^{3}-\nu^{3}-\lambda \mu \nu\right) \sigma+\left(\nu \lambda-\mu^{2}\right)\left(\nu \mu-\lambda^{2}\right)=0 .
$$

It may be remarked that if $\sigma=0$, then $a$ or $b$ vanishes; and therefore, from the original equations, $\nu \lambda-\mu^{2}=0$, or $\nu \mu-\lambda^{2}=0$, which agrees with the result afforded by the foregoing equation in $\sigma$. Again, if $\sigma=\nu$, then $c=0$; and therefore, from the original equations, $\nu^{2}-\lambda \mu=0$. The left-hand side of the equation in $\sigma$, writing therein $\sigma=\nu$, should therefore contain the factor $\nu^{2}-\lambda \mu$; its value in fact is $\nu^{4}-2 \lambda \mu \nu^{2}+\lambda^{2} \mu^{2}$, or $\left(\nu^{2}-\lambda \mu\right)^{2}$.

If in the original equations we write $a=\frac{x}{z}, b=\frac{y}{z}$, the equations become

$$
\begin{aligned}
& x^{2}+c y z-\lambda z^{2}=0, \\
& y^{2}+c z x-\mu z^{2}=0, \\
& \left(c^{2}-\nu\right) z^{2}+x y=0,
\end{aligned}
$$

which are three homogeneous equations of the second order; from which, if the variables $x, y, z$ are eliminated, we have the required equation in $c$. And it would not, I think, be difficult, from the known formula for the general case, to deduce the foregoing result corresponding to the very particular case which is here in question.

2, Stone Buildings, W.C., September 25, 1860. 